\title{
A Systematic Review and Meta-Analysis Examining Pneumonia-Associated Mortality in Dementia
}

\author{
Norine C. Foley ${ }^{a, b}$, Rebecca H. Affoo ${ }^{g}$ Ruth E. Martin ${ }^{a, c-e}$ \\ aSchool of Communication Sciences and Disorders, Elborn College, Departments of \\ bPhysical Medicine and Rehabilitation, ${ }^{\mathrm{C}}$ Physiology and Pharmacology and \\ dOtolaryngology, and eNeuroscience Program, Schulich School of Medicine and Dentistry, \\ Western University, and f Department of Foods and Nutrition, Brescia University College, \\ and ${ }^{9}$ Graduate Program of Health and Rehabilitation Sciences, London, Ont., Canada
}

\section{Key Words}

Alzheimer's disease $\cdot$ Cause of death · Epidemiology

\begin{abstract}
Background: Although it is generally accepted that deaths associated with pneumonia are more common in patients with dementia, no comprehensive reviews on the subject have previously been published. Summary: Relevant studies were identified through a literature search of the PubMed, EMBASE, Scopus, and ISI Web of Science databases for publications up to August 2013. Studies were included if (1) a group of adult subjects with dementia and a (comparison) group composed of subjects without dementia were included, (2) the cause(s) of death was/were reported, and (3) pneumonia was identified as one of the possible causes of death. The occurrence of death due to pneumonia associated with dementia was expressed as an odds ratio (OR) with $95 \%$ confidence interval (CI). Thirteen studies were included. The odds of death resulting from pneumonia were significantly increased for persons with any form of dementia compared with those without dementia (OR $=2.22,95 \%$ CI 1.44-3.42, $p<$ 0.001). In a subgroup analysis, using the results from 8 studies that restricted inclusion to persons with Alzheimer's disease, the odds of death resulting from pneumonia were also significantly higher (OR $=1.70,95 \%$ CI 1.12-2.58, $p=0.013)$. Key Messages: The odds of pneumonia-associated mortality were increased more than 2-fold for patients with dementia.
\end{abstract}


Foley et al:: A Systematic Review and Meta-Analysis Examining Pneumonia-Associated Mortality in Dementia

\section{Introduction}

Dementia is an umbrella term, which encompasses a group of related neurodegenerative disorders such as Alzheimer's disease (AD), Lewy body dementia, frontotemporal dementia, vascular dementia, and Parkinson's disease dementia. The disease is a progressive disorder that impairs memory, thinking, and the ability to perform activities of daily living and is associated with premature disability and death in developed countries [1,2]. Persons with dementia tend to die at an earlier age and for different reasons compared with persons without dementia [3-5]. While cardiovascular diseases and neoplasms are more frequent causes of death in the general population, persons with dementia tend to die more often from infections, including pneumonia [6]. In fact, pneumonia has been cited as the most common cause of death in patients with $\mathrm{AD}$ [7-10]. Although it is generally accepted that deaths due to pneumonia are more common in patients with dementia, compared with those without dementia, there are contradictory reports $[4,11,12]$, and no systematic reviews on the subject have been published. Therefore, the objectives of this study were to conduct a systematic review and meta-analysis to determine whether patients with dementia are more likely to die of pneumonia as the immediate or underlying cause, and if so, to estimate the magnitude of the increased risk.

\section{Methods}

Relevant studies were identified through a literature search encompassing the period from 1985 to August 2013. The following databases were searched: PubMed (MEDLINE), EMBASE, Scopus, and ISI Web of Science. Search terms varied slightly across databases but included the terms 'dementia' OR 'Alzheimer's dementia' AND 'pneumonia' OR 'aspiration pneumonia' AND mortality OR 'cause of death' as either MeSH terms, key words, or subject headings (see Appendix for details of the search terms used in each of the databases). Where possible, the searches were limited to 'Human', 'All adults; 19+ years', and the English language. Hand searching of the bibliographies of the included studies was conducted to identify potential articles not identified in the search. The inclusion criteria were broad to capture as many potentially relevant articles as possible.

Studies that included a group of patients with dementia and a control group, reporting the causes of death, were sought. The diagnosis of dementia could be made clinically before death (in population-based studies) or after death based on autopsy reports or death certificates (in studies that used hospital-based samples). Similarly, subjects in the control or the comparison group could include any group of individuals who did not have dementia.

\section{Inclusion Criteria}

Studies of any design were included if the following criteria were met: (1) the sample included a group of adult participants ( $\geq 19$ years) who were identified with dementia (AD, vascular dementia, or non-specified dementia) and a (comparison) group composed of subjects without dementia; (2) the presence of dementia was identified through a standardized process that was described - or, following death, by autopsy - or it had been documented in the medical chart; (3) the cause of death, based on either death certificates or autopsy reports, was reported; (4) pneumonia, aspiration pneumonia, or bronchopneumonia was identified as one of the possible causes of death, and (5) the study was published in the English language.

\section{Exclusion Criteria}

Studies in which all, or a portion of the participants, suffered from Huntington's disease, Parkinson's disease, Creutzfeldt-Jakob disease, or AIDS-related dementia were excluded, as were studies in which vague terms such as chronic brain syndrome were used or where the results of the dementia subgroup were not reported separately when the study's sample included mixed diagnoses.

Titles and abstracts identified by the literature search were first screened for eligibility by two authors (N.C.F. and R.H.A.). If the eligibility remained in question, the full article was retrieved and reviewed. Disagreements were resolved through consensus. Data pertaining to participant characteristics, criteria used to 
identify/diagnose dementia, and deaths associated with pneumonia, aspiration pneumonia, or bronchopneumonia were extracted from each eligible trial by one reviewer (N.C.F.) and verified for accuracy by a second author (R.H.A.). The odds ratio (OR) and 95\% confidence interval (CI) of pneumonia-associated mortality given the presence of dementia were estimated for each study using the software Comprehensive Meta-Analysis (version 2, 2007; Biostat Inc., Englewood, N.J., USA), and the results were pooled. The preplanned subgroup analysis included AD and sample origin (population or hospital based). When provided, the most adjusted estimates of risks and odds of death reported in the individual studies were used in pooled analyses; otherwise, the raw proportions of persons with and those without dementia who died due to pneumonia were used. A random-effects model was used to account for true variation in effect size and sampling error (i.e., between- and within-study variability).

\section{Results}

\section{Literature Search Results}

The literature search results and the reasons for exclusions are presented in figure 1 . The initial search returned 568 results, after the removal of duplicates. Following the review of titles and abstracts, 23 potentially relevant studies remained for detailed examination, 12 of which met all inclusion criteria. One additional study was retrieved through hand searching, leaving 13 studies in total for review [3, 4, 6, 11-20].

A summary of the details of all included studies is presented in tables 1 and 2 . The reporting details in one study, an extension of a previous study [10] and reported in abstract form, were limited [15]. Eight of these studies included participants who were selected from the general population. In these studies, incident cases of dementia were identified based on standardized criteria. Participants with and those without dementia were followed up over time, and the cause(s) of death was/were established during the course of the study's followup period or after all the study participants had died $[3,4,11,16-20]$. The average lengths of follow-up varied between studies from 4 to 10 years. In 5 studies, the samples were composed of groups of patients with and patients without dementia who had died following admission to the hospital $[6,12-15]$. In 4 of these studies, both the cases and the controls were drawn from the same institution; in the fifth study, persons from the general population formed the comparison group [6]. With one exception [14], the confirmation of dementia was verified post mortem based on brain pathology, and the cause(s) of death also was/were established based on autopsy.

Participants diagnosed with AD exclusively composed the dementia group in 4 studies $[3,4,14,16]$. In the remaining studies, participants with $\mathrm{AD}$ represented $>50 \%(\mathrm{n}=5$ studies $)$ $[11,13,17,18,20]$ or $<50 \%$ ( $n=2$ studies) $[6,12]$ of the dementia cases, or the breakdown of dementia types was not reported [15, 19]. Other types of dementia included vascular dementia, mixed dementia, and unspecified, 'other' dementia. In 5 of the population-based studies, the participants' mean age at baseline was reported for both the dementia and the comparison group, and in all cases, those with dementia were significantly older $[4,11,17$, $18,20]$. In the 2 hospital-based studies where age was reported, those with dementia were significantly older at the time of hospital death $[12,13]$. There was no difference in the sex distribution between groups in 2 studies $[11,19]$, while significantly more females were included in the dementia group in 4 studies $[4,13,17,18]$. The severity/stage of dementia at study entry was reported in 2 population-based studies $[11,18]$. In one case, the majority of participants suffered from mild or very mild disease [11], while most participants in the other study had moderate or severe forms of dementia [18].

Most of the studies classified mortality into general categories, which included cardiovascular disease, cerebrovascular diseases, neoplasm (cancer), accident, pulmonary disorders, digestive tract disorders, other neurological disorders, pneumonia, and 'other'. Immediate, 
Foley et al.: A Systematic Review and Meta-Analysis Examining Pneumonia-Associated Mortality in Dementia

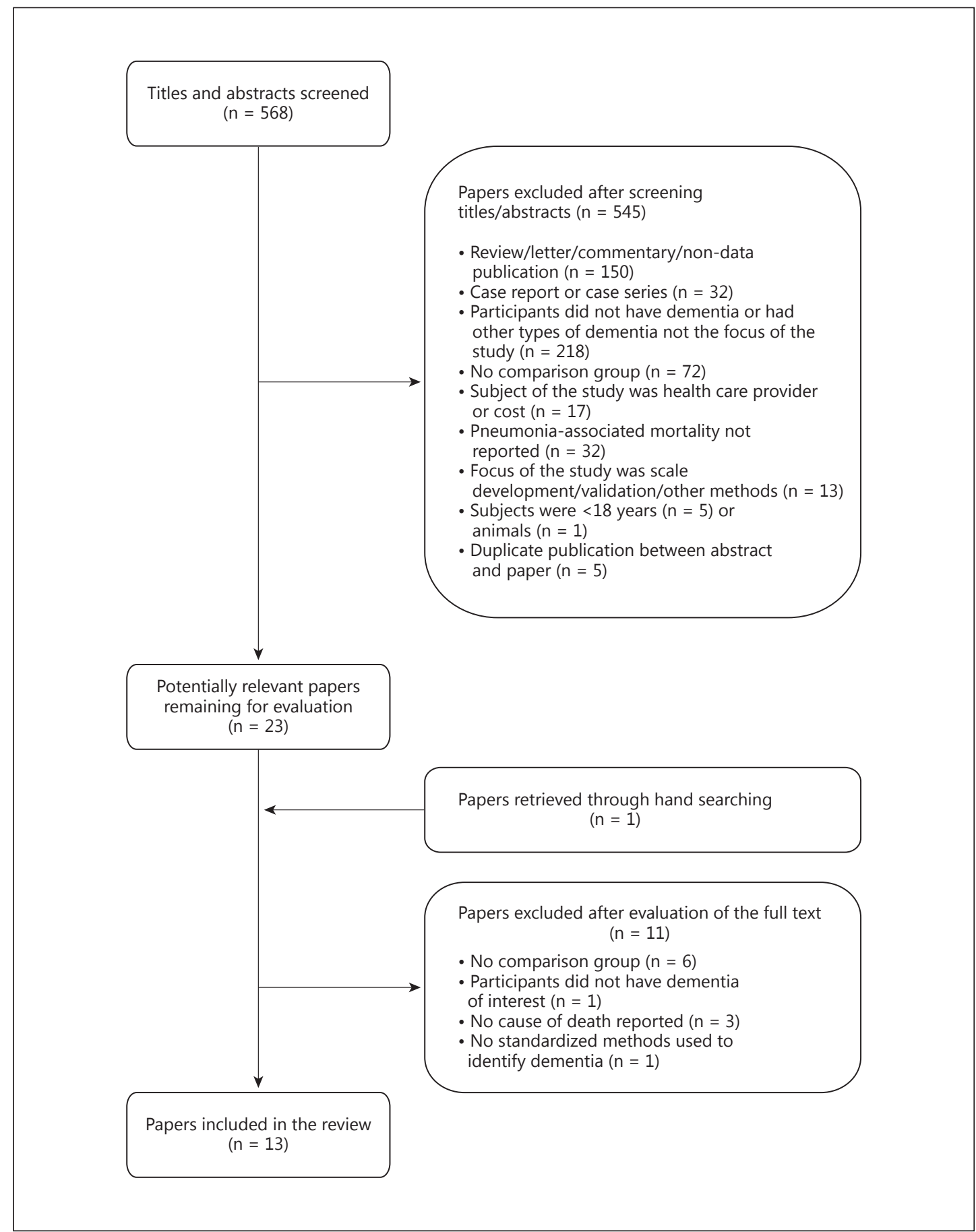

Fig. 1. Literature search results.

underlying, and contributory significant conditions of death were reported in 2 studies [4, 16], while immediate or underlying or contributory causes of death were reported in 1 study [3]. The immediate cause of death only was reported in 3 studies $[12,17,19]$, while the underlying cause of death was reported in 3 other studies $[6,18,20]$. It was unclear whether the immediate or the underlying cause of death was reported in the remaining studies. 
Dementia

Cognitive Disorders

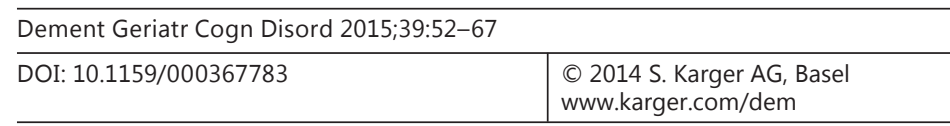

Foley et al.: A Systematic Review and Meta-Analysis Examining Pneumonia-Associated Mortality in Dementia

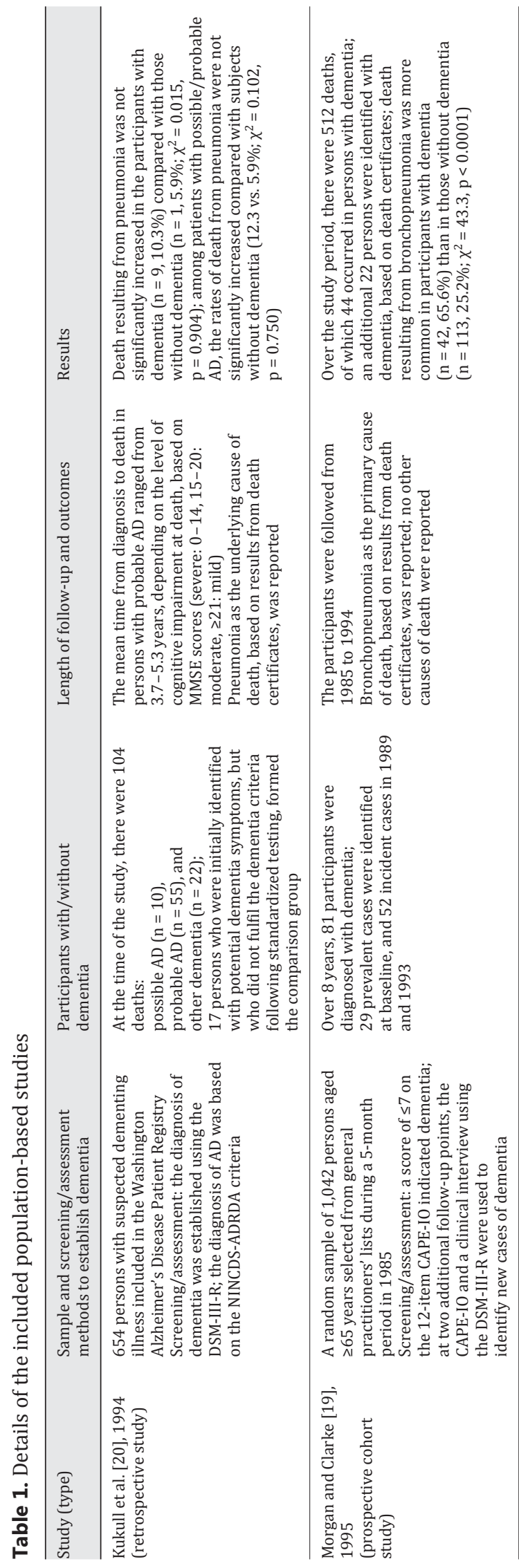

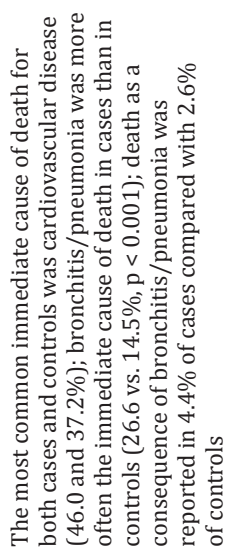

)

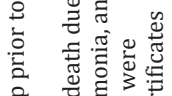

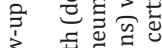

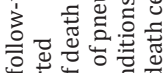

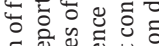

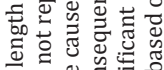

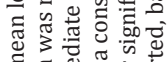

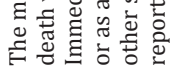

这蓠。

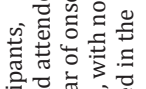

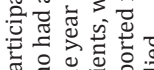

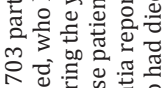

记

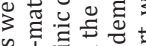

능

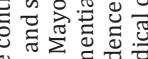

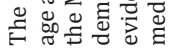

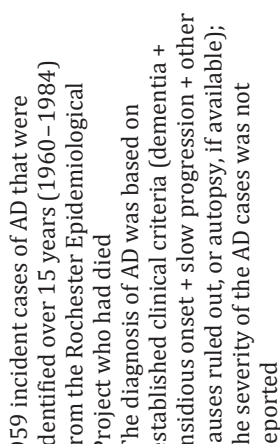

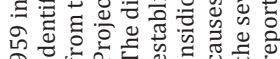

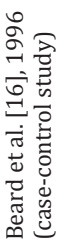

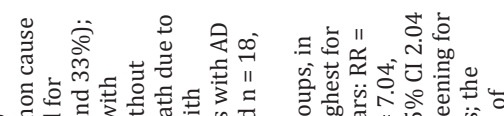

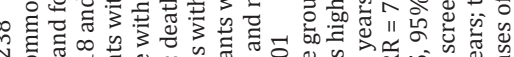

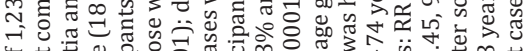

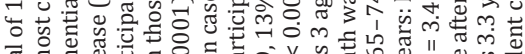

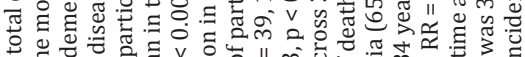

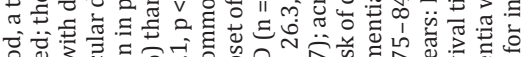

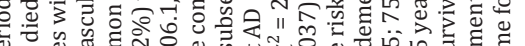

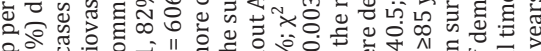

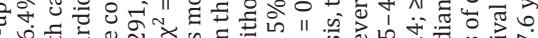

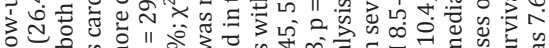

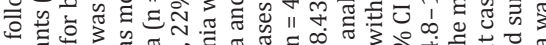

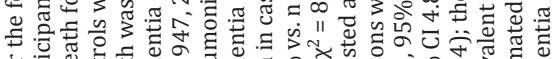

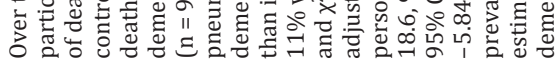

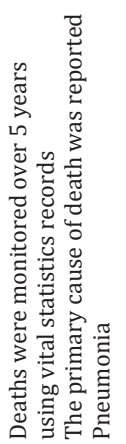

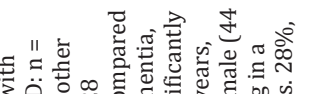
30ิ

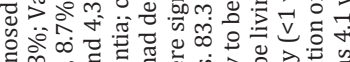

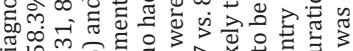

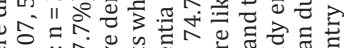

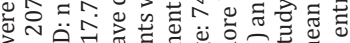

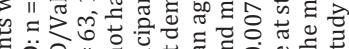

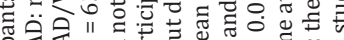

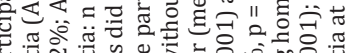

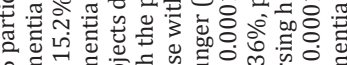

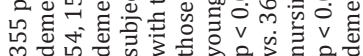

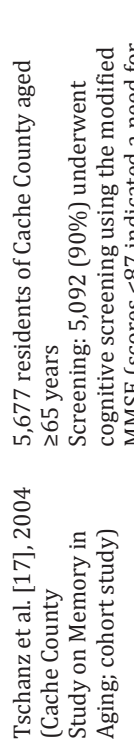




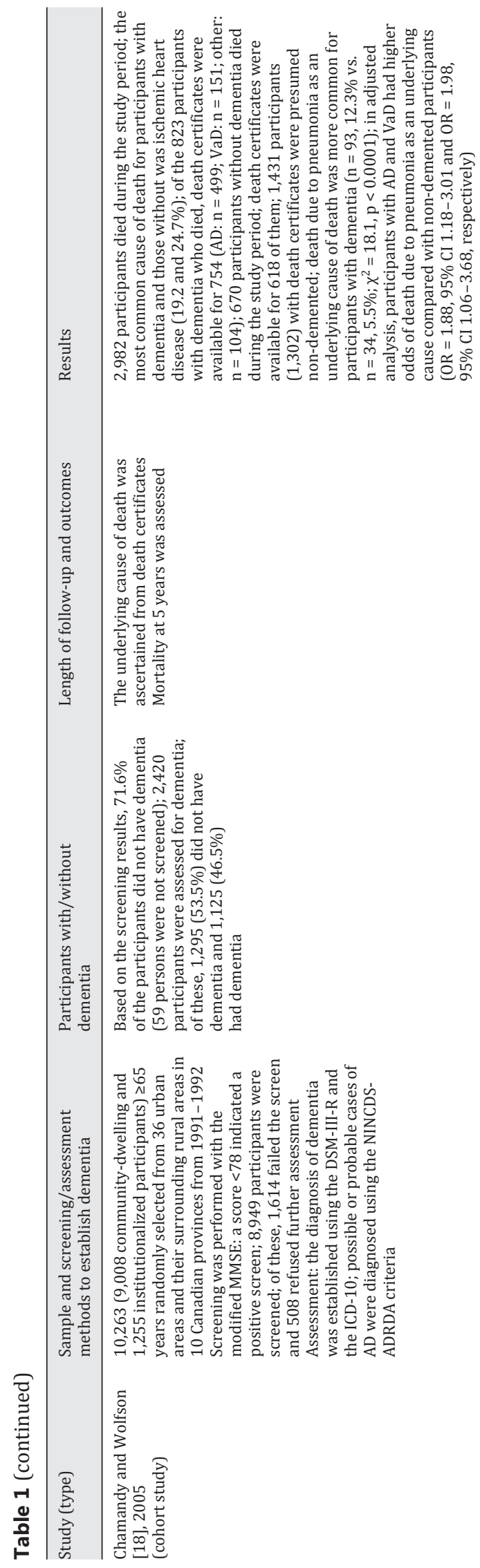

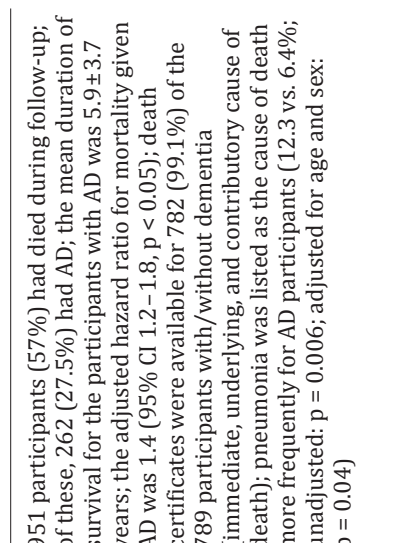

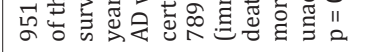
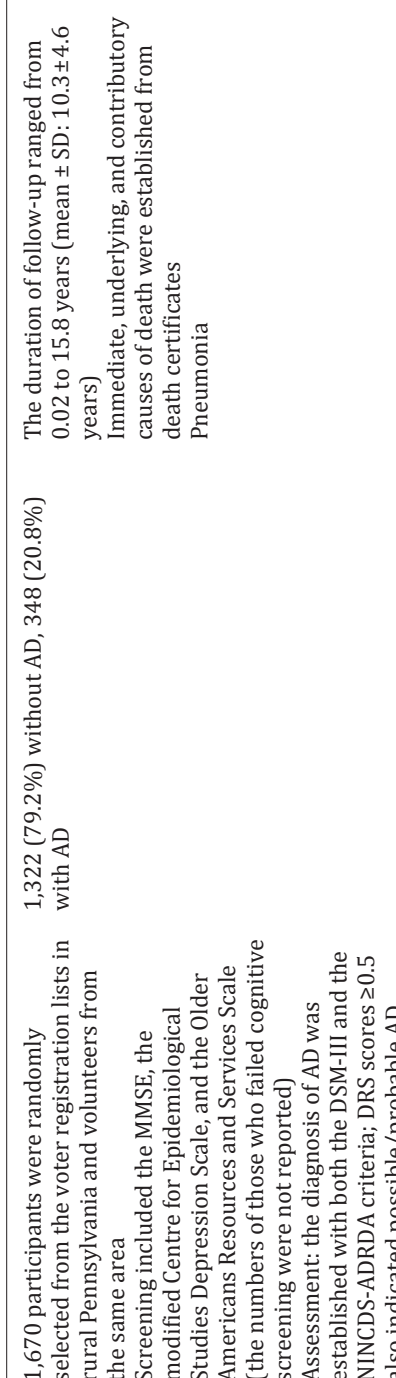

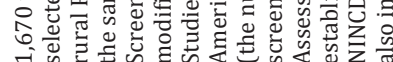

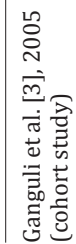

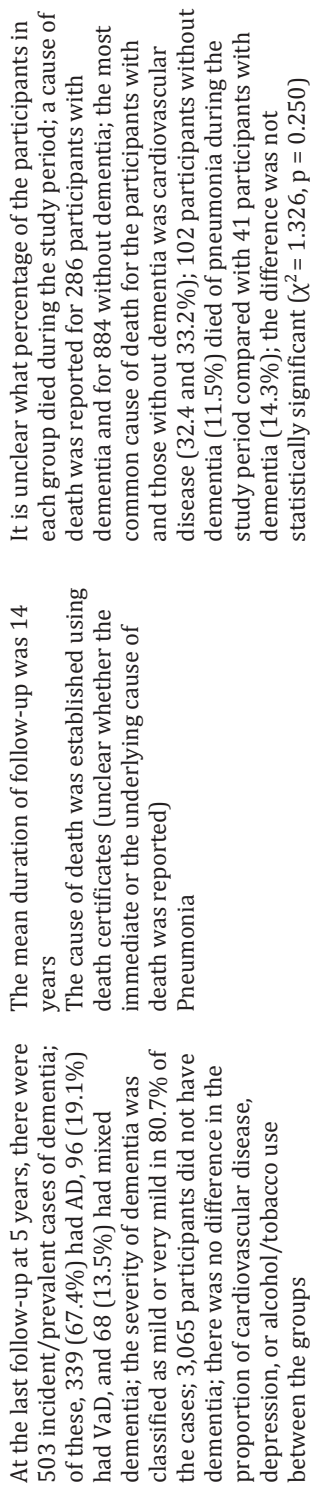

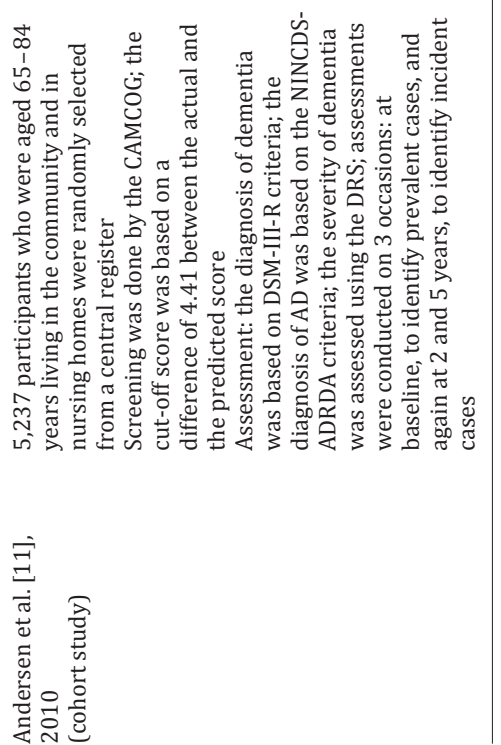



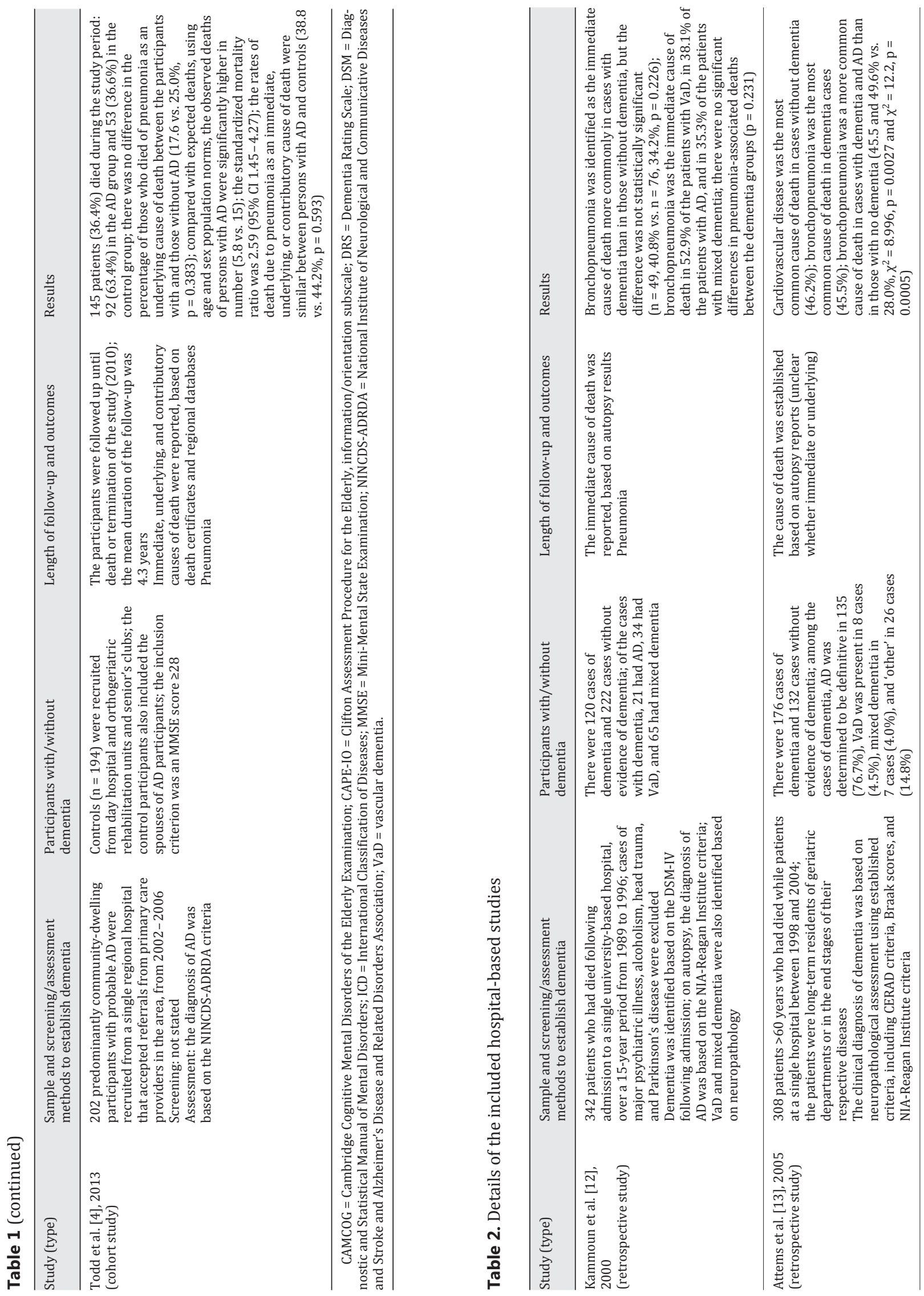
Dementia

Cognitive Disorders
Dement Geriatr Cogn Disord 2015;39:52-67

DOI: $10.1159 / 000367783$

Foley et al.: A Systematic Review and Meta-Analysis Examining Pneumonia-Associated Mortality in Dementia

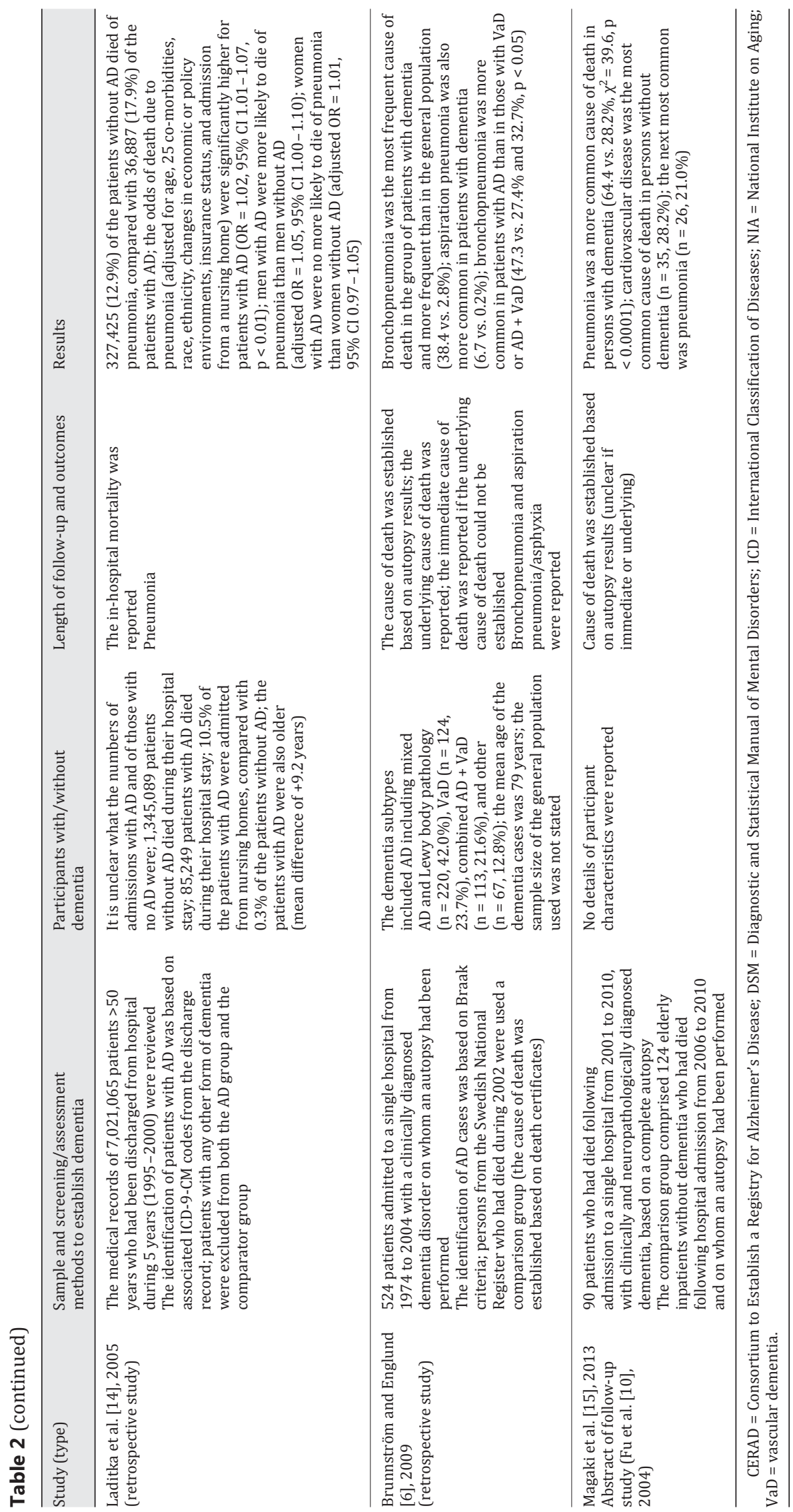


Foley et al:: A Systematic Review and Meta-Analysis Examining Pneumonia-Associated Mortality in Dementia

Pneumonia - or what were regarded as similar terms, including aspiration pneumonia, bronchopneumonia, or bronchitis/pneumonia - as a cause of death was reported significantly more often among patients with dementia than among patients without dementia in the majority of studies [3, 6, 13-19]. In 3 of the 4 large prospective population-based studies, a significantly higher percentage of participants with dementia died as a result of pneumonia, ranging from 12.3 to $14.3 \%$, compared with $5-11.5 \%$ of patients in the control (no dementia) groups $[3,17,18]$. In hospital-based studies, the percentages of both patients with and those without dementia who died from pneumonia were much higher (38-64.4\% vs. $21.0-34.2 \%$ ) $[6,12,13,15]$, and significantly more persons with dementia died as a result of the condition. When reported, a small percentage of patients' deaths were attributed to dementia or AD (2.4-4.0\%), although it was much higher in 1 study, accounting for $23.5 \%$ of deaths among patients in the dementia group [4]. The most common causes of death of patients without dementia were cardiovascular and cerebrovascular diseases (including stroke and myocardial infarction) and cancer.

Data from 12 studies were available for pooled analysis. The odds of pneumonia-associated mortality were significantly increased for persons with any form of dementia compared with those without dementia (OR $=2.22,95 \%$ CI 1.44-3.42, p < 0.001) (fig. 2). In the subgroup analysis, using the results from 8 studies that restricted inclusion to persons with AD or in which the results from this group were reported separately, the pooled odds of death resulting from pneumonia were also significantly higher (OR $=1.70,95 \%$ CI 1.12-2.58, $\mathrm{p}=0.013$ ) (fig. 3). The odds of pneumonia-associated mortality were higher in studies using populationbased samples (OR $=2.32,95 \%$ CI $1.15-3.53, \mathrm{p}<0.001)$ than in studies in which data from hospital-based samples were used (OR $=2.12,95 \%$ CI 0.99-4.52, $\mathrm{p}=0.053$ ).

\section{Discussion}

The results of this study indicated that the odds of pneumonia-associated mortality were increased more than 2 -fold in patients with dementia. In studies using hospital-based samples, the reasons for admission, the living arrangements prior to admission, the presence of co-morbid conditions, the stage of dementia, swallowing status, and other salient medical information were not reported. Similarly, in studies that had recruited patients from the community and followed them up for years, the locations and circumstances surrounding their deaths were unknown, although it may assumed that the majority of patients were admitted to the hospital or were institutionalized at the time of death. As a result, it was unclear whether the increased rates of death from pneumonia were disproportionately due to infection that was contracted in the advanced stage of dementia or whether, alternatively, patients who were admitted to the hospital in the milder stage of disease progression for unrelated medical or surgical reasons developed pneumonia as a complication during their hospital stay and died. Evidence suggests that excess mortality rates could have been linked to both causes. It has been reported that patients who develop dementia are hospitalized more frequently than those without dementia [21-23] and are often admitted on an emergent basis $[9,24]$. The odds of hospital admission given the presence of pre-existing dementia have been reported to be 1.41 [22] and 3.68 times higher [25] and are known to rise with an increasing number of co-morbid conditions [26]. Following an initial hospitalization, patients with dementia are also more likely to be re-admitted within 3 months of discharge [27]. While it might be expected that increased hospital admission rates occur with increasing frequency in patients with more advanced disease progression, few studies have examined this relationship. Compared with persons without dementia, the odds of hospital admission were reported to be 2.3 times greater in patients with severe $A D$, but they were not raised signifi- 


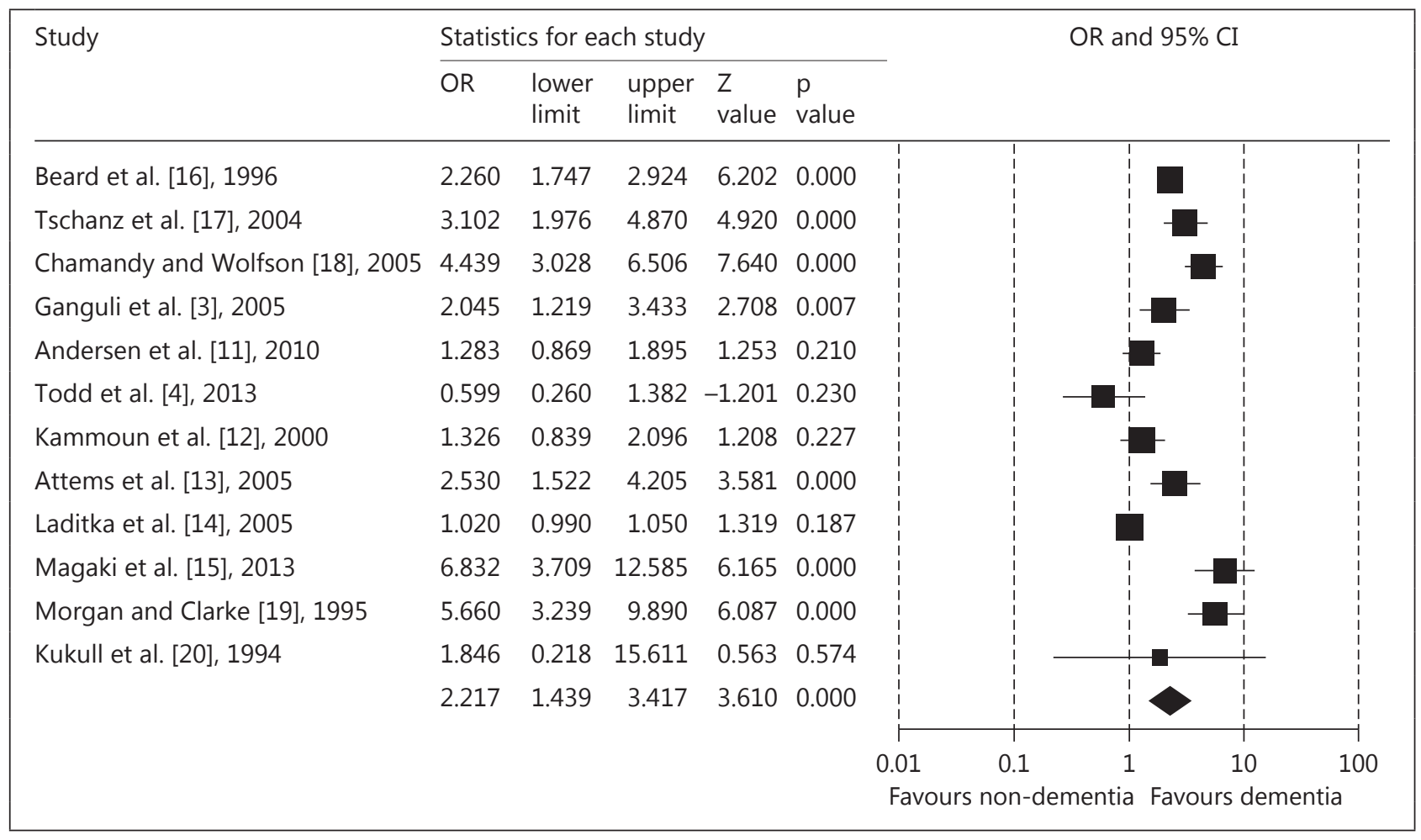

Fig. 2. Forest plot of pneumonia-associated mortality.

cantly in patients with mild-to-moderate $\mathrm{AD}$, even when accounting for co-morbid medical conditions [28]; however, in a study that included community-dwelling individuals, the majority of whom were in the mild stage of AD with low co-morbidity, $66 \%$ were hospitalized at least once during a median follow-up of 2.2 years [26].

A higher proportion of patients with dementia are known to be hospitalized with potentially preventable conditions including pneumonia, urinary tract infections, and dehydration [22]. A portion of the increased risk of hospitalization among persons with dementia has been directly attributed to an increase in the number of cases of pneumonia [28]. The odds of admission due to pneumonia were reported to be 1.50 [21] and 1.88 [22] times greater in patients with dementia than in a control group. Pneumonia has also been reported as an in-hospital complication more frequently for patients with dementia. The risk of developing pneumonia was significantly elevated in patients with dementia admitted for medical and surgical reasons - by $37 \%$ and $66 \%$, respectively [29]. In a large sample restricted to surgical admissions, the presence of dementia was associated with a significant increase in the development of a number of post-operative complications, including pneumonia (OR $=2.44,95 \%$ CI 2.31-2.58) [30]. In addition to a greater frequency of hospitalizations related to dementia, the age- and sex-adjusted odds of in-hospital mortality from all causes have been reported to be 1.77 [31] and 2.1 [5] times higher for patients with dementia. The odds of death are further increased if patients experience at least 1 adverse clinical event during hospitalization [5].

There are several mechanisms through which patients with dementia may be especially vulnerable to the development of pneumonia. Pneumonia can be precipitated by an episode of aspiration associated with dysphagia [32, 33], a common sequela of dementia [34-36]. While dysphagia is most commonly reported in patients with advanced dementia [32,33], it has also been observed in patients in the earlier, milder stages of disease progression [37]. 
Foley et al:: A Systematic Review and Meta-Analysis Examining Pneumonia-Associated Mortality in Dementia

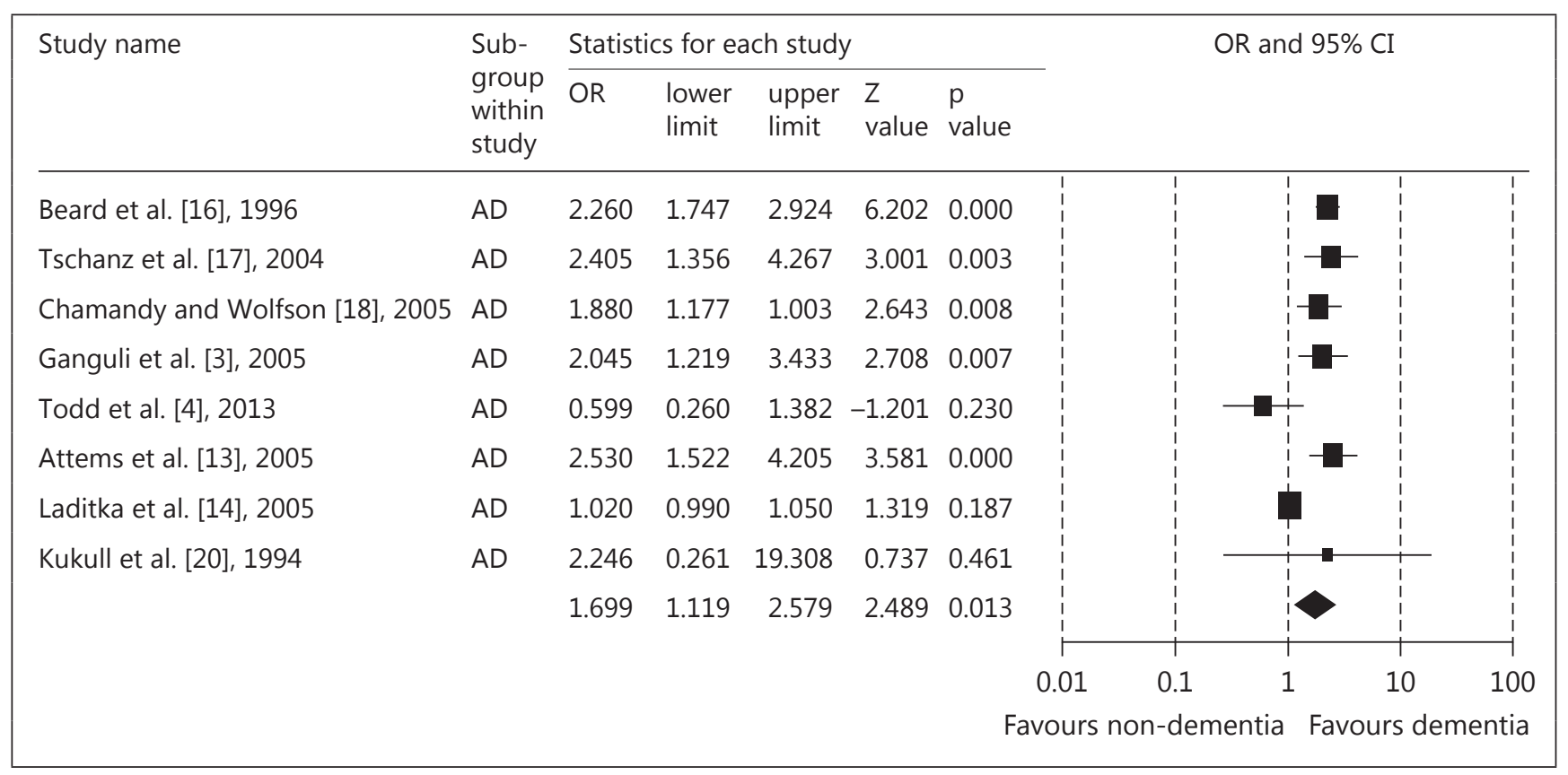

Fig. 3. Forest plot of pneumonia-associated mortality (AD).

Gastro-oesophageal reflux may also contribute to the development of pneumonia, particularly when enteral feeding tubes are used in the management of dysphagia [38]. While these devices are intended as a lower-risk means of feeding persons with severe dysphagia, their use has been associated with an increased incidence of aspiration pneumonia in advanced dementia [39].

Although the potential contribution of swallowing problems and gastro-oesophageal reflux could not be investigated in the present review, one author noted that death due to bronchopneumonia and aspiration pneumonia was common, and that the presence of dysphagia was often noted in the clinical record [6]. The presence of a swallowing disorder has previously been correlated with death from pneumonia in persons with advanced dementia [40], and aspiration has been cited as a major cause of death in individuals fed with percutaneous endoscopic gastrostomy tubes [38]. To help reduce the risk of potentially avoidable cases of pneumonia associated with dysphagia and gastro-oesophageal reflux, several measures can be taken. Closer attention to oral care and hygiene, diet modifications to facilitate swallowing in the presence of dysphagia, and education for caregivers who may assist with oral feeding may help to reduce the risk of pneumonia associated with dysphagiarelated aspiration. In the more advanced stages of the disease, when pneumonia may be an indicator of approaching death [41], aggressive measures such as the use of tube feeding, intended to help reduce the dangers of aspiration, may not be appropriate [42].

It has also been suggested that the diagnosis and treatment of pneumonia may be delayed in patients whose ability to communicate is impaired, especially in the early stages when patients may be afebrile [30]. A bidirectional relationship between cognitive function and pneumonia has recently been demonstrated, whereby declines in cognitive status increased patients' risk of the development of pneumonia and the rate of cognitive decline accelerated following an episode of pneumonia [43]. Impairment of the inflammatory response system, resulting in higher levels of circulating cytokines and pro-inflammatory markers, in turn leading to neuroinflammation, was suggested as a possible mechanism. Side effects caused by 
some types of medications such as neuroleptics may also contribute to pharyngeal dysphagia [44]. Additional, non-specific factors that may have contributed to the excess rates of death from pneumonia in persons with dementia, and which are common in frail seniors, include a reduced ability to clear secretions, impaired immune function [10], increased co-morbidity, and reduced mobility [45].

Potential sources of heterogeneity between studies that may have affected the overall effect size estimate included the cause of death that was reported (underlying vs. immediate), the method used to establish the cause of death (autopsy vs. death certificate), the dementia type and severity, and the study sample (hospital vs. population-based cases and controls). Due to a lack of reporting details, not all sources of variability could be explored. Type of dementia has been suggested as a potential effect modifier, with an increased risk of pneumonia-associated mortality noted among patients with $\operatorname{AD}[13,46]$. In the present study, when the analysis was restricted to 8 studies that included patients with AD only, the pooled estimate was $33 \%$ lower than the overall result (OR $=1.670$ vs. 2.22$)$. The difference in estimates may be accounted for, in part, by the higher risk of pneumonia-related death associated with vascular dementia (data not shown). Although only 2 studies were included in this subgroup, the effect size was very large in one of them [17]. Suh et al. [47] reported that sensory dysfunction was seen more frequently in patients with $A D$, while patients with vascular dementia had more difficulties with bolus formation and mastication of semi-solid food. The incidence of both aspiration and silent aspiration was significantly higher among patients with vascular dementia (47 vs. $13.3 \%, p=0.024$ and 32 vs. $0 \%, p=0.011$, respectively), which potentially may have placed them at a higher risk of pneumonia. Although studies examining other types of dementia were not identified, dysphagia has been documented in Lewy body dementia, Parkinson's disease dementia, and frontotemporal dementia, and all are associated with aberrant eating behaviour as well as motor and sensory disturbances, potentially increasing the risk of aspiration leading to pneumonia [48-52].

An increasing severity of dementia has been associated with an increased risk of death from all causes, including pneumonia. Dementia severity was found to be an independent predictor of pneumonia-associated mortality within the first week of diagnosis and at 3 months [53]. The relationship between baseline dementia severity and risk of death from pneumonia was explored in only 1 of the prospective studies included in the present review [18]. Compared with patients in a mild dementia group, severe dementia was associated with a non-significant increased odds of death (OR $=1.99,95 \% \mathrm{CI} 0.88-4.50)$. In the same study, there was no association between the severity of dementia at the time of death and pneumonia-related mortality, using the proxy measures of baseline severity and duration of disease. In contrast, Kukull et al. [20] reported that deaths from pneumonia as an underlying cause were more frequent in patients with severe dementia than in those with mild dementia (22.2 vs. 9.1\%). Neoplasms, stroke, and other vascular diseases were the most commonly reported causes of death for patients with mild dementia, while pneumonia and death from dementia itself were the most common underlying causes of death in patients with severe impairment. Since the severity (or stage) of dementia was not reported at the time of death in any of the other studies, its effect as a potential source of variability could not be examined further.

Speculating as to the variability in effect size associated with using autopsy versus death certificates to determine the cause of death is challenging. Poor agreement between causes of death based on death certificates and those based on autopsy reports has previously been noted, and information on death certificates is known to be less accurate. Pneumonia as a cause of death has been under-reported when autopsy results have been compared with death certificates in persons with dementia $[7,8]$. Pneumonia was identified as the primary cause of death in $47 \%$ of cases based on death certificates and in $57 \%$ of cases based on 
autopsy results and was missed on 11 of 53 death certificates [8]. We included 9 studies that relied on death certificate or registry data to establish a cause of death. While the number of true cases in which pneumonia had been the cause of death was unknown in these studies, it is possible that they were under-estimated, although a bias favouring one group (dementia or control) is unlikely. Pneumonia-associated mortality may have been under-reported in studies that only reported the underlying cause of death, given that pneumonia is more likely to be reported as an immediate cause. In 1 of the included studies, the first-listed causes of death on parts $1 \mathrm{a}, \mathrm{b}$, and $\mathrm{c}$ of the death certificates were all reported [16]. Pneumonia was identified as an immediate cause of death in both cases and controls 6 times more frequently than as an underlying cause.

\section{Limitations}

In some of the included studies, an estimation of the effect size was calculated using crude (unadjusted) data, likely resulting in an over-estimation of the effect size. This fact may help to explain why, contrary to expectation, the odds of pneumonia-related death were $9 \%$ higher in studies including population-based samples, even though patients in hospital-based studies were perceived to be at higher risk. In only 1 of the hospital-based studies was the analysis adjusted for potentially confounding variables [14], while adjustments were made in 3 of the population-based studies $[3,16,18]$. In the remaining studies, when reported, a greater proportion of patients at higher risk, including females and those of more advanced age, were represented in the dementia group. It is possible that, despite our best effort, potentially relevant articles were missed in the literature search.

In summary, the results from 13 studies suggest that the odds of pneumonia-associated mortality were significantly increased for persons with any form of dementia compared with those without dementia. The causes of the increased risk may include aspiration - associated with dysphagia - as well as non-specific factors, which are common in frail seniors and further increased in the terminal stages of dementia. Although it was not possible to determine at what stage of the disease process pneumonia developed, it is likely that a portion of the excess mortality rates in dementia could be prevented by appropriate early diagnosis and management.

\section{Appendix}

Literature Search Strategy

PubMed $(\mathrm{n}=179)$

(('Dementia'[MeSH]) OR ('Alzheimer Disease'[MeSH])) AND (('Pneumonia'[MeSH]) OR ('Pneumonia, Aspiration' $[\mathrm{MeSH}])$ )

Limits: English language, adults ( $\geq 19$ years)

EMBASE $(\mathrm{n}=276)$

(Dementia/ or Alzheimer disease/) AND (bacterial pneumonia/ or community acquired pneumonia/ or aspiration pneumonia/ or pneumonia/ or hospital acquired pneumonia/) AND (cause of death'/ or mortality)

Limits: English language, human

Scopus $(\mathrm{n}=403)$

KEY(dementia OR 'Disease, Alzheimer' OR 'Alzheimer's Disease' OR 'Disease, Alzheimer's' OR 'Alzheimer Type Dementia' OR 'Senile Dementia, Alzheimer Type' OR 'Dementia, Primary Senile Degenerative' OR 'Dementia, Senile' OR 'Dementias, Senile' OR 'Senile Dementia' OR 'Senile Dementias' OR 'Primary Senile Degenerative Dementia' OR 'Alzheimer Type Senile Dementia' OR 'Dementia, Presenile' OR 'Dementias, Presenile' OR 'Presenile Dementia' OR 'Presenile Dementias' OR 'Alzheimer Disease, Late Onset' OR 'Late Onset Alzheimer Disease' OR 'Alzheimer's Disease, Focal Onset' OR 'Focal Onset Alzheimer's Disease' OR 
'Acute Confusional Senile Dementia' OR 'Senile Dementia, Acute Confusional' OR 'Alzheimer Disease, Early Onset' OR 'Presenile Alzheimer Dementia' OR 'Alzheimer Dementia, Presenile' OR 'Dementia, Presenile Alzheimer' OR 'Early Onset Alzheimer Disease' OR 'Dementias, Vascular' OR 'Vascular Dementias' OR 'Vascular Dementia' OR 'Vascular Dementia, Acute Onset' OR 'Acute Onset Vascular Dementia' OR 'Subcortical Vascular Dementia' OR ‘Dementia, Subcortical Vascular' OR 'Dementias, Subcortical Vascular' OR 'Subcortical Vascular Dementias' OR 'Vascular Dementia, Subcortical' OR 'Vascular Dementias, Subcortical' OR 'Dementia, Multi Infarct' OR 'Dementias, Multi-Infarct' OR 'Multi-Infarct Dementias' OR 'Multi-Infarct Dementia' OR 'Multi Infarct Dementia' OR 'Dementia, Multiinfarct' OR 'Dementias, Multiinfarct' OR 'Multiinfarct Dementia' OR 'Multiinfarct Dementias' OR 'Dementia Multi-Infarct' OR 'Dementia Multi Infarct' OR 'Dementia MultiInfarcts' OR 'Multi-Infarct, Dementia' OR 'Multi-Infarcts, Dementia' OR 'Dementias, Frontotemporal' OR 'Frontotemporal Dementias' OR 'Frontotemporal Lobe Dementia' OR 'Dementia, Frontotemporal Lobe' OR 'Dementias, Frontotemporal Lobe’ OR ‘Frontotemporal Lobe Dementias' OR 'Lewy Body Dementia' OR 'Lewy Body Disease, Cortical' OR ‘Dementia, Lewy Body' OR 'Lewy Body Type Senile Dementia' OR ‘Cortical Lewy Body Disease' OR 'Diffuse Lewy Body Disease' OR 'Lewy Body Disease, Diffuse') AND KEY(Pneumoni* OR 'Pulmonary Inflammation' OR 'Inflammation, Pulmonary' OR 'Inflammations, Pulmonary' OR 'Pulmonary Inflammations' OR ‘Lung Inflammation' OR 'Inflammations, Lung' OR ‘Lung Inflammations' OR 'Lobar Pneumonia' OR 'Pneumonia, Lobar' OR 'Aspiration Pneumonias' OR 'Pneumonias, Aspiration' OR 'Aspiration Pneumonia' OR 'Acid Aspiration Syndrome' OR 'Acid Aspiration Syndromes' OR Bronchopneumonia* OR 'Bronchial Pneumonia' OR 'Bronchial Pneumonias' OR 'Pneumonia, Bronchial' OR 'Pneumonias, Bronchial') AND KEY(Mortality OR 'Mortality, In-Hospital' OR 'In-Hospital Mortalities' OR 'Mortalities, In-Hospital' OR 'In-Hospital Mortality' OR 'Mortality, Hospital' OR 'Mortalities, Hospital' OR 'Mortalities, In-house' OR 'In-house Mortalities' OR 'In-house Mortality' OR ‘Mortalities, In house' OR 'Mortality, In-house' OR 'Mortality, Inhospital' OR 'Hospital Mortalities' OR 'In Hospital Mortalities' OR 'Inhospital Mortalities' OR 'Mortalities, Inhospital' OR 'In Hospital Mortality' OR 'Hospital Mortalities, In' OR 'Hospital Mortality, In' OR 'Mortalities, In Hospital' OR 'Mortality, In Hospital' OR 'Inhospital Mortality' OR 'Cause of Death')

Limits: English language

ISI Web of Science $(\mathrm{n}=188)$

TS = (Pneumoni* OR 'Pulmonary Inflammation' OR 'Inflammation, Pulmonary' OR 'Inflammations, Pulmonary' OR 'Pulmonary Inflammations' OR 'Lung Inflammation' OR 'Inflammations, Lung' OR 'Lung Inflammations' OR 'Lobar Pneumonia' OR 'Pneumonia, Lobar' OR 'Aspiration Pneumonias' OR 'Pneumonias, Aspiration' OR 'Aspiration Pneumonia' OR ‘Acid Aspiration Syndrome' OR 'Acid Aspiration Syndromes' OR Bronchopneumonia* OR ‘Bronchial Pneumonia' OR 'Bronchial Pneumonias' OR 'Pneumonia, Bronchial’ OR 'Pneumonias, Bronchial') AND TS = (dementia OR 'Disease, Alzheimer' OR 'Alzheimer's Disease' OR 'Disease, Alzheimer's' OR 'Alzheimer Type Dementia' OR 'Senile Dementia, Alzheimer Type' OR 'Dementia, Primary Senile Degenerative' OR 'Dementia, Senile' OR 'Dementias, Senile' OR 'Senile Dementia' OR 'Senile Dementias' OR 'Primary Senile Degenerative Dementia' OR 'Alzheimer Type Senile Dementia' OR 'Dementia, Presenile' OR 'Dementias, Presenile' OR 'Presenile Dementia' OR 'Presenile Dementias' OR 'Alzheimer Disease, Late Onset' OR 'Late Onset Alzheimer Disease' OR 'Alzheimer's Disease, Focal Onset' OR 'Focal Onset Alzheimer's Disease' OR 'Acute Confusional Senile Dementia' OR 'Senile Dementia, Acute Confusional' OR 'Alzheimer Disease, Early Onset' OR 'Presenile Alzheimer Dementia' OR 'Alzheimer Dementia, Presenile' OR ‘Dementia, Presenile Alzheimer' OR 'Early Onset Alzheimer Disease' OR 'Dementias, Vascular' OR 'Vascular Dementias' OR 'Vascular Dementia' OR 'Vascular Dementia, Acute Onset' OR ‘Acute Onset Vascular Dementia' OR 'Subcortical Vascular Dementia' OR 'Dementia, Subcortical Vascular' OR 'Dementias, Subcortical Vascular' OR 'Subcortical Vascular Dementias' OR 'Vascular Dementia, Subcortical' OR 'Vascular Dementias, Subcortical' OR 'Dementia, Multi Infarct' OR 'Dementias, Multi-Infarct' OR 'Multi-Infarct Dementias' OR 'Multi-Infarct Dementia' OR 'Multi Infarct Dementia' OR 'Dementia, Multiinfarct' OR 'Dementias, Multiinfarct' OR 'Multiinfarct Dementia' OR 'Multiinfarct Dementias' OR 'Dementia Multi-Infarct' OR 'Dementia Multi Infarct' OR 'Dementia Multi-Infarcts' OR 'Multi-Infarct, Dementia' OR 'Multi-Infarcts, Dementia' OR 'Dementias, Frontotemporal' OR 'Frontotemporal Dementias' OR 'Frontotemporal Lobe Dementia' OR 'Dementia, Frontotemporal Lobe' OR 'Dementias, Frontotemporal Lobe' OR 'Frontotemporal Lobe Dementias' OR 'Lewy Body Dementia' OR 'Lewy Body Disease, Cortical' OR 'Dementia, Lewy Body' OR 'Lewy Body Type Senile Dementia' OR ‘Cortical Lewy Body Disease’ OR ‘Diffuse Lewy Body Disease’ OR 'Lewy Body Disease, Diffuse') AND TS = ('cause of death' OR mortality)

Limits: English language

Total unique hits: $\mathrm{n}=568$ 


\section{Acknowledgments}

The authors would like to thank Mrs. Heather Campbell (Librarian, Brescia University College) for her assistance conducting the literature search and Sheryl Tang for her assistance in manuscript preparation. The study was funded by the Canadian Institute for Health Research (grant No. 99467).

\section{Disclosure Statement}

The authors have no conflicts of interest to declare.

\section{References}

1 Michaud CM, McKenna MT, Begg S, Tomijima N, Majmudar M, Bulzacchelli MT, Ebrahim S, Ezzati M, Salomon JA, Kreiser JG, Hogan M, Murray CJ: The burden of disease and injury in the United States 1996. Popul Health Metr 2006; 4:11.

$\longrightarrow 2$ Murray CJ, Lopez AD: Alternative projections of mortality and disability by cause 1990-2020: Global Burden of Disease Study. Lancet 1997;349:1498-1504.

3 Ganguli M, Dodge HH, Shen C, Pandav RS, DeKosky ST: Alzheimer disease and mortality: a 15-year epidemiological study. Arch Neurol 2005;62:779-784.

4 Todd S, Barr S, Passmore AP: Cause of death in Alzheimer's disease: a cohort study. QJM 2013;106:747-753.

5 Marengoni A, Corrao S, Nobili A, Tettamanti M, Pasina L, Salerno F, Iorio A, Marcucci M, Bonometti F, Mannucci PM: In-hospital death according to dementia diagnosis in acutely ill elderly patients: the REPOSI study. Int J Geriatr Psychiatry 2011;26:930-936.

6 Brunnström HR, Englund EM: Cause of death in patients with dementia disorders. Eur J Neurol 2009;16:488492.

7 Burns A, Jacoby R, Luthert P, Levy R: Cause of death in Alzheimer's disease. Age Ageing 1990;19:341-344.

8 Keene J, Hope T, Fairburn CG, Jacoby R: Death and dementia. Int J Geriatr Psychiatry 2001;16:969-974.

-9 Natalwala A, Potluri R, Uppal H, Heun R: Reasons for hospital admissions in dementia patients in Birmingham, UK, during 2002-2007. Dement Geriatr Cogn Disord 2008;26:499-505.

10 Fu C, Chute DJ, Farag ES, Garakian J, Cummings JL, Vinters HV: Comorbidity in dementia: an autopsy study. Arch Pathol Lab Med 2004;128:32-38.

11 Andersen K, Lolk A, Martinussen T, Kragh-Sørensen P: Very mild to severe dementia and mortality: a 14-year follow-up - The Odense Study. Dement Geriatr Cogn Disord 2010;29:61-67.

-12 Kammoun S, Gold G, Bouras C, Giannakopoulos P, McGee W, Herrmann F, Michel JP: Immediate causes of death of demented and non-demented elderly. Acta Neurol Scand Suppl 2000;176:96-99.

13 Attems J, König C, Huber M, Lintner F, Jellinger KA: Cause of death in demented and non-demented elderly inpatients; an autopsy study of 308 cases. J Alzheimers Dis 2005;8:57-62.

14 Laditka JN, Laditka SB, Cornman CB: Evaluating hospital care for individuals with Alzheimer's disease using inpatient quality indicators. Am J Alzheimers Dis Other Demen 2005;20:27-36.

15 Magaki S, Boon-Unge K, Su K, Yong W, Khanlou N, Vinters H: Comorbidity in dementia: an update of an autopsy study (poster). 89th Annu Meet Am Assoc Neuropathol, Charleston, 2013, p 580.

-16 Beard CM, Kokmen E, Sigler C, Smith GE, Petterson T, O’Brien PC: Cause of death in Alzheimer's disease. Ann Epidemiol 1996;6:195-200.

-17 Tschanz JT, Corcoran C, Skoog I, Khachaturian AS, Herrick J, Hayden KM, Welsh-Bohmer KA, Calvert T, Norton MC, Zandi P, Breitner JC: Dementia: the leading predictor of death in a defined elderly population: the Cache County Study. Neurology 2004;62:1156-1162.

18 Chamandy N, Wolfson C: Underlying cause of death in demented and non-demented elderly Canadians. Neuroepidemiology 2005;25:75-84.

19 Morgan K, Clarke D: To what extent is dementia underreported on British death certificates? Int J Geriatr Psychiatry 1995;10:987-990.

20 Kukull WA, Brenner DE, Speck CE, Nochlin D, Bowen J, McCormick W, Teri L, Pfanschmidt ML, Larson EB: Causes of death associated with Alzheimer disease: variation by level of cognitive impairment before death. J Am Geriatr Soc 1994;42:723-726.

21 Zhao Y, Kuo TC, Weir S, Kramer MS, Ash AS: Healthcare costs and utilization for Medicare beneficiaries with Alzheimer's. BMC Health Serv Res 2008;8:108.

22 Phelan EA, Borson S, Grothaus L, Balch S, Larson EB: Association of incident dementia with hospitalizations. JAMA 2012;307:165-172.

23 Zuliani G, Galvani M, Sioulis F, Bonetti F, Prandini S, Boari B, Guerzoni F, Gallerani M: Discharge diagnosis and comorbidity profile in hospitalized older patients with dementia. Int J Geriatr Psychiatry 2012;27:313-320. 
Foley et al:: A Systematic Review and Meta-Analysis Examining Pneumonia-Associated Mortality in Dementia

24 Nourhashemi F, Andrieu S, Sastres N, Ducasse JL, Lauque D, Sinclair AJ, Albarede JL, Vellas BJ: Descriptive analysis of emergency hospital admissions of patients with Alzheimer disease. Alzheimer Dis Assoc Disord 2001;15:21-25.

25 Bynum JP, Rabins PV, Weller W, Niefeld M, Anderson GF, Wu AW: The relationship between a dementia diagnosis, chronic illness, Medicare expenditures, and hospital use. J Am Geriatr Soc 2004;52:187-194.

-26 Rudolph JL, Zanin NM, Jones RN, Marcantonio ER, Fong TG, Yang FM, Yap L, Inouye SK: Hospitalization in community-dwelling persons with Alzheimer's disease: frequency and causes. J Am Geriatr Soc 2010;58: 1542-1548.

-27 Draper B, Karmel R, Gibson D, Peut A, Anderson P: The Hospital Dementia Services Project: age differences in hospital stays for older people with and without dementia. Int Psychogeriatr 2011;23:1649-1658.

28 Albert SM, Costa R, Merchant C, Small S, Jenders RA, Stern Y: Hospitalization and Alzheimer's disease: results from a community-based study. J Gerontol A Biol Sci Med Sci 1999;54:M267-M271.

29 Bail K, Berry H, Grealish L, Draper B, Karmel R, Gibson D, Peut A: Potentially preventable complications of urinary tract infections, pressure areas, pneumonia, and delirium in hospitalised dementia patients: retrospective cohort study. BMJ Open 2013;3:e002770.

30 Hu CJ, Liao CC, Chang CC, Wu CH, Chen TL: Postoperative adverse outcomes in surgical patients with dementia: a retrospective cohort study. World J Surg 2012;36:2051-2058.

-31 Guijarro R, San Roman CM, Gomez-Huelgas R, Villalobos A, Martin M, Guil M, Martinez-Gonzalez MA, Toledo JB: Impact of dementia on hospitalization. Neuroepidemiology 2010;35:101-108.

-32 Feinberg MJ, Ekberg 0, Segall L, Tully J: Deglutition in elderly patients with dementia: findings of videofluorographic evaluation and impact on staging and management. Radiology 1992;183:811-814.

-33 Horner J, Alberts MJ, Dawson DV, Cook GM: Swallowing in Alzheimer's disease. Alzheimer Dis Assoc Disord 1994;8:177-189.

-34 Sue EE: Dysphagia and aspiration pneumonia in older adults. J Am Acad Nurse Pract 2010;22:17-22.

-35 van der Maarel-Wierink CD, Vanobbergen JN, Bronkhorst EM, Schols JM, de Baat C: Meta-analysis of dysphagia and aspiration pneumonia in frail elders. J Dent Res 2011;90:1398-1404.

-36 Pace CC, McCullough GH: The association between oral microorgansims and aspiration pneumonia in the institutionalized elderly: review and recommendations. Dysphagia 2010;25:307-322.

-37 Humbert IA, McLaren DG, Kosmatka K, Fitzgerald M, Johnson S, Porcaro E, Kays S, Umoh EO, Robbins J: Early deficits in cortical control of swallowing in Alzheimer's disease. J Alzheimers Dis 2010;19:1185-1197.

-38 Suzuki Y, Tamez S, Murakami A, et al: Survival of geriatric patients after percutaneous endoscopic gastrostomy in Japan. World J Gastroenterol 2010;16:5084-5091.

39 Finucane TE, Christmas C, Travis K: Tube feeding in patients with advanced dementia: a review of the evidence. JAMA 1999;282:1365-1370.

40 Chouinard J, Lavigne E, Villeneuve C: Weight loss, dysphagia, and outcome in advanced dementia. Dysphagia 1998;13:151-155.

41 Mitchell SL, Teno JM, Kiely DK, Shaffer ML, Jones RN, Prigerson HG, Volicer L, Givens JL, Hamel MB: The clinical course of advanced dementia. N Engl J Med 2009;361:1529-1538.

-42 American Geriatrics Society Ethics Committee; Clinical Practice and Models of Care Committee: American Geriatrics Society feeding tubes in advanced dementia position statement. J Am Geriatr Soc 2014;62:15901593.

-43 Shah FA, Pike F, Alvarez K, Angus D, Newman AB, Lopez O, Tate J, Kapur V, Wilsdon A, Krishnan JA, Hansel N, Au D, Avdalovic M, Fan VS, Barr RG, Yende S: Bidirectional relationship between cognitive function and pneumonia. Am J Respir Crit Care Med 2013;188:586-592.

44 Balzer KM: Drug-induced dysphagia. Int J MS Care 2000;2:40-50.

45 Bosch X, Formiga F, Cuerpo S, Torres B, Roson B, Lopez-Soto A: Aspiration pneumonia in old patients with dementia. Prognostic factors of mortality. Eur J Intern Med 2012;23:720-726.

46 Thomas BM, Starr JM, Whalley LJ: Death certification in treated cases of presenile Alzheimer's disease and vascular dementia in Scotland. Age Ageing 1997;26:401-406.

47 Suh MK, Kim H, Na DL: Dysphagia in patients with dementia: Alzheimer versus vascular. Alzheimer Dis Assoc Disord 2009;23:178-184.

48 Yamamoto T, Kobayashi Y, Murata M: Risk of pneumonia onset and discontinuation of oral intake following videofluorography in patients with Lewy body disease. Parkinsonism Relat Disord 2010;16:503-506.

-49 Shinagawa S, Adachi H, Toyota Y, Mori T, Matsumoto I, Fukuhara R, Ikeda M: Characteristics of eating and swallowing problems in patients who have dementia with Lewy bodies. Int Psychogeriatr 2009;21:520-525.

50 Bine JE, Frank EM, McDade HL: Dysphagia and dementia in subjects with Parkinson's disease. Dysphagia 1995; 10:160-164.

-51 Langmore SE, Olney RK, Lomen-Hoerth C, Miller BL: Dysphagia in patients with frontotemporal lobar dementia. Arch Neurol 2007;64:58-62.

52 Ikeda M, Brown J, Holland AJ, Fukuhara R, Hodges JR: Changes in appetite, food preference, and eating habits in frontotemporal dementia and Alzheimer's disease. J Neurol Neurosurg Psychiatry 2002;73:371-376.

53 van der Steen JT, Ooms ME, Mehr DR, van der Wal G, Ribbe MW: Severe dementia and adverse outcomes of nursing home-acquired pneumonia: evidence for mediation by functional and pathophysiological decline. J Am Geriatr Soc 2002;50:439-448. 